\title{
Una propuesta de tríada diagnóstica a propósito de un caso de oclusión por fitobezoar
}

\section{A proposed diagnostic triad for a case of phytobezoar occlusion Uma proposta para uma tríade de diagnóstico em um caso de oclusão fitobezoar}

\author{
doi) http://dx.doi.org/10.35954/SM2020.39.2.6 \\ Martín Varela Vega a (iD https://orcid.org/0000-0003-2376-2844 \\ Micaela Mandacen b (iD) https://orcid.org/0000-0002-0368-2372 \\ Catalina González c (i) https://orcid.org/0000-0003-2929-5263 \\ Rodrigo Demolín c (D) https://orcid.org/0000-0002-0039-9467 \\ Iván Trostchansky c (i) https://orcid.org/0000-0002-9023-3750 \\ Santiago Cubas a (i) https://orcid.org/0000-0001-6026-5316 \\ Carlos Barberousse ${ }^{a}$ (iD) https://orcid.org/0000-0001-9982-9369
(a) Asistente de Cátedra. Clínica Quirúrgica B, Hospital de Clínicas Dr. Manuel Quintela, Facultad de Medicina, Universidad de la República.
(b) Cátedra de Imagenología, Hospital de Clínicas Dr. Manuel Quintela, Facultad de Medicina, Universidad de la República.
(c) Departamento de Emergencias, Hospital Central de las Fuerzas Armadas.

\begin{abstract}
RESUMEN
Los fitobezoares son depósitos persistentes de fibra vegetal no digerible en el tubo digestivo, habitualmente localizados en el estómago, aunque pueden migrar a otras partes del intestino. Suelen manifestarse como una oclusión intestinal completa o incompleta. El diagnóstico es difícil de realizar en el preoperatorio, siendo la mayoría de las veces un hallazgo intraoperatorio. En caso de topografía intestinal, el tratamiento quirúrgico es la regla. Al punto de partida de un caso y de revisión de literatura sobre el tema proponemos una tríada que puede favorecer el diagnóstico preoperatorio.
\end{abstract}

PALABRAS CLAVE: Bezoares; Obstrucción intestinal.

\begin{abstract}
Phytobezoars are persistent deposits of non-digestible plant fiber in the digestive tract, usually located in the stomach, although they can migrate to other parts of the intestine. They usually manifest as complete or incomplete intestinal occlusion. The diagnosis is difficult to make preoperatively, being most of the time an intraoperative finding. In case of intestinal topography, surgical treatment is the rule. On the basis of a case and a review of the literature on the subject, we propose a triad that can help the preoperative diagnosis.
\end{abstract}

KEY WORDS: Bezoars; Intestinal Obstruction. 


\section{RESUMO}

Os fitobezoares são depósitos persistentes de fibra vegetal não digerível no trato digestivo, geralmente localizados no estômago, embora possam migrar para outras partes do intestino. Eles geralmente se manifestam como oclusão intestinal completa ou incompleta. O diagnóstico é difícil de fazer no pré-operatório, sendo na maioria das vezes um achado intra-operatório. No caso da topografia intestinal, o tratamento cirúrgico é a regra. Com base em um caso e uma revisão da literatura sobre o assunto, propomos uma tríade que pode ajudar o diagnóstico pré-operatório.

PALAVRAS CHAVE: Bezoares; Obstrução Intestinal.

\section{INTRODUCCIÓN}

Los bezoares son depósitos persistentes de material no digerible en el tubo digestivo, habitualmente localizados en el estómago, aunque pueden migrar a otras partes del intestino. Generalmente se componen de fibra vegetal (fitobezoares), pelo (tricobezoares), medicamentos (fármacobezoares) (1). En el caso de los fitobezoares, son factores predisponentes la ingesta abundante de fibra vegetal (acelga, espinaca, naranja, uvas con cáscara, etc.) en el contexto de un paciente con dentadura incompleta, generalmente añoso. Los bezoares gástricos son especialmente frecuentes en pacientes gastrectomizados en donde hay una disminución del tamaño de la cámara gástrica, una alteración de la motilidad y un pH más alcalino de lo habitual $(2,3)$.

En ocasiones pueden migrar al intestino delgado, siendo el sitio más frecuente de oclusión la válvula íleo cecal (4).

Suelen manifestarse como una oclusión intestinal completa o incompleta. El diagnóstico es difícil de realizar en el preoperatorio, siendo la mayoría de las veces un hallazgo intraoperatorio (5). La tomografía computada (TC) puede mostrar elementos de oclusión de delgado, característicamente con una transición entre asas delgadas no distendidas y un asa dilatada que en su interior tiene contenido intestinal con burbujas aéreas (signo de la miga de pan o de las heces) (6).

Las opciones terapéuticas abarcan un amplio rango, desde el tratamiento médico con reposo digestivo, endoscópico, digestión enzimática con dilución de celulasa o ingesta de Coca Cola ${ }^{\circledR}$ cuando son de topografía gástrica, mientras que cuando están localizados en el intestino delgado el tratamiento más frecuente es la cirugía (7). A continuación presentamos un caso clínico de resolución en nuestro servicio.

\section{CASO CLÍNICO}

Mujer de 73 años con antecedente de cesárea abdominal hace muchos años, ingresada en el Hospital Central de las Fuerzas Armadas por neumonía, con buena respuesta al tratamiento antibiótico. Antes del alta comienza con dolor abdominal tipo cólico, vómitos y detención del tránsito. Se coloca una sonda nasogástrica, con retención de líquido intestinal. Abdomen distendido y ruidos hidroaéreos aumentados en timbre y en frecuencia. Se solicita TC que evidencia distención del intestino delgado hasta fosa ilíaca izquierda, donde se observa sitio de transición asa delgada dilatada - chata (figura 1). Con diagnóstico presuntivo de oclusión de delgado por bridas se inicia tratamiento médico con reposo digestivo y de gravitación mediante sonda nasogástrica.

Dado que la paciente no restablece el tránsito digestivo con el tratamiento médico, se decide realizar cirugía, que es de inicio laparoscópica pero debe convertirse a laparotómica ante dificultad de exposición por la gran distención abdominal. En la misma se constata en íleon distal masa endoluminal de consistencia blanda y escasamente móvil (figura 2). 


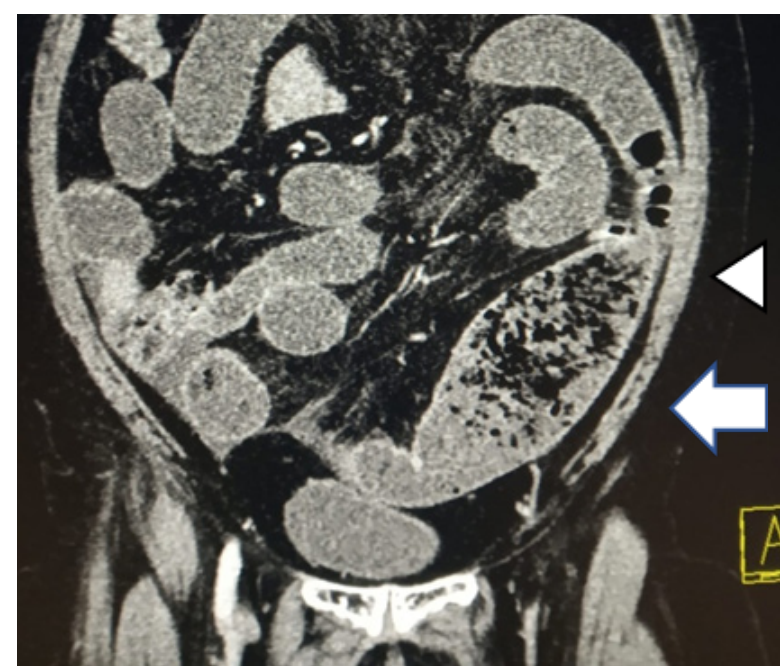

Figura 1. Tomografía computada con contraste iv. Reconstrucción coronal. Se visualizan elementos de oclusión intestinal de delgado; observamos asas delgadas distendidas, con punto de transición (cabeza de flecha); a nivel de fosa iliaca izquierda se evidencia el "signo de la miga de pan" (flecha blanca).

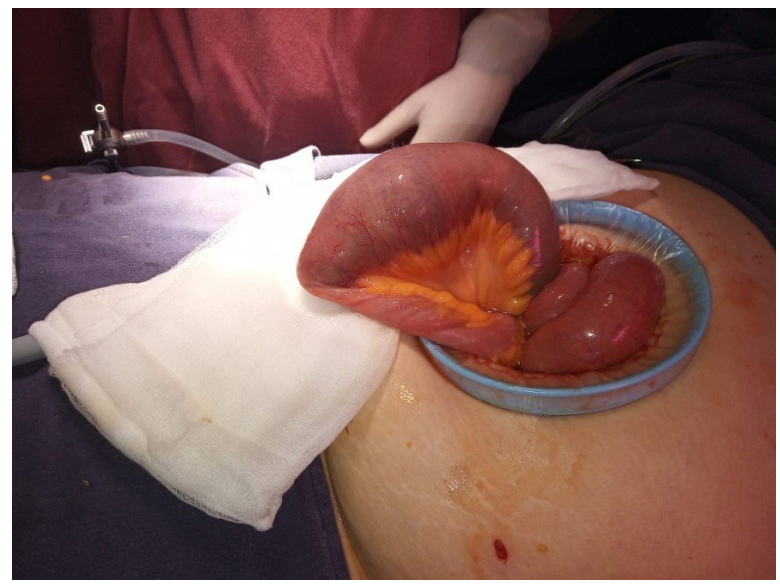

Figura 2. Cirugía. Evisceración de asa de delgado, donde se observa transición determinada por masa intraluminal.

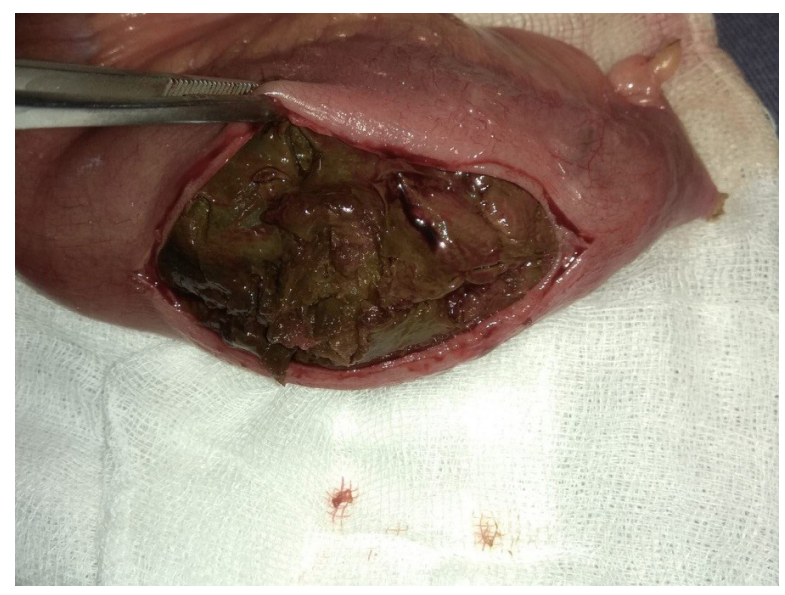

Figura 3. Pieza abierta con fitobezoar impactado.
Se realiza resección del asa y anastomosis término terminal. Una vez abierta la pieza se constata fitobezoar impactado (figura 3).

Buena evolución postoperatoria con alta al cuarto día.

\section{DISCUSIÓN}

El fitobezoar es una rara causa de oclusión intestinal, que se ve más frecuente en personas ancianas, con dentadura incompleta, y el antecedente de ingesta de verduras de hoja verde o frutas con cáscara. Radiológicamente pueden tener elementos de oclusión intestinal sumado a la imagen característica de "la miga de pan" o "signo de las heces" dentro del delgado. Este signo radiológico se da por la existencia de burbujas de aire entre las fibras vegetales, lo que da la imagen característica similar a las heces colónicas (8). En el caso de tener un diagnóstico preoperatorio certero, cuando la localización es gástrica pueden ser tratados mediante endoscopía, o con la ingesta de celulasa para la digestión enzimática de las fibras vegetales (9).

Incluso hay descripciones de tratamiento exitoso con Coca Cola ${ }^{\circledR}$. Esto es más factible en el caso de localización gástrica del mismo (7). Pero en otras ocasiones el diagnóstico es operatorio, y en ese caso puede resolverse mediante enterotomía con extracción de las fibras vegetales.

\section{CONCLUSIÓN}

La oclusión intestinal por fitobezoar es rara, y su diagnóstico es difícil cuando no hay un bajo umbral de sospecha por parte del equipo médico, llevando en ocasiones a realizar un diagnóstico tardío. Puede ser útil tener en cuenta la tríada de sospecha de oclusión por fitobezoar: paciente anciano con dentadura incompleta, con oclusión de delgado y signo radiológico de la miga de pan.

DECLARACIÓN DE CONFLICTOS DE INTERESES: Los autores no reportan ningún conflicto de interés. El estudio se realizó con recursos propios de los autores y/o la institución a la que representan. 


\section{REFERENCIAS}

(1) Lacoma Latre EM, Sánchez Lalana E, Saudí Moro S. Fitobezoar intestinal. Imagen Diag 2017; 8(1):21-23.

doi: 10.1016/j.imadi.2016.02.004

(2) Rider JA, Foresti-Lorente RF, Garrido J, Puletti EJ; Rider DL, King AH, et al.

Gastric bezoars: treatment and prevention. Am J Gastroenterol 1984; 79(5):357-359.

PMID: 6720657

(3) Cabo Navarro D, Fort López-Barajas JM, Mesa Manteca J, Lecube Torelló A.

Fitobezoar en remanente gástrico tras cirugía bariátrica. Med Clin (Barc) 2009; 132(5):200.

doi: 10.1016/j.medcli.2008.09.008

(4) Toccaceli S, Donfrancesco A, Stella LP, Diana M, Dandolo R, Di Schino C.

Shall bowel obstruction caused by phytobezoar. Case report. G Chir 2005; 26(5):218-220.

PMID: 16184707

(5) Soriano Pérez MJ, Serrano Carrillo de Albornoz JL, Marín Montín I, Merino Rumín MC.

Obstrucción intestinal secundaria a fitobezoar.

Med Clin (Barc) 2007; 129(16):640.

doi: 10.1016/s0025-7753(07)72949-4
(6) Quiroga S, Alvarez-Castells A, Sebastia C, Pallisa E, Barluenga E.

Small bowel obstruction secondary to bezoar: CT diagnosis. Abdom Imaging 1997; 22(3):315-317. doi: $10.1007 / \mathrm{s} 002619900198$

(7) Martínez de Juan F, Martínez-Lapiedra C, Picazo V. Phytobezoar dissolution with Coca-Cola. Gastroenterol Hepatol 2006; 29(5):291-293. doi: $10.1157 / 13087468$

(8) Lazarus DE, Slywotsky C, Bennett GL, Megibow AJ, Macari M.

Frequency and relevance of the "small-bowel feces" sign on CT in patients with small-bowel obstruction. AJR Am J Roentgenol 2004; 183(5):1361-1366. doi: 10.2214/ajr.183.5.1831361

(9) Fernández Morató J, Ilzarbe Sánchez J, Bessa Caserras J, Mateu de Antonio J. Tratamiento con celulasa en 3 casos de fitobezoares de gran tamaño.

Farm Hosp 2009; 33(2):100-3. PMID: 19480798 